\title{
Some new results and inequalities for subsequences of Nörlund logarithmic means of Walsh-Fourier series
}

\author{
David Baramidze ${ }^{1,2}$, Lars-Erik Persson ${ }^{2,3^{*}}$, Harpal Singh² and George Tephnadze
}

"Correspondence:
larserik6pers@gmail.com
${ }^{2}$ UiT The Arctic University of
Norway, P.O. Box 385, N-8505,
Narvik, Norway
${ }^{3}$ Department of Mathematics and
Computer Science, Karlstad
University, 65188 Karlstad, Sweden
Full list of author information is
available at the end of the article

available at the end of the article

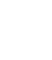

\begin{abstract}
We prove that there exists a martingale $f \in H_{p}$ such that the subsequence $\left\{L_{2^{n}} f\right\}$ of Nörlund logarithmic means with respect to the Walsh system are not bounded from the martingale Hardy spaces $H_{p}$ to the space weak $-L_{p}$ for $0<p<1$. We also prove that for any $f \in L_{p}, p \geq 1, L_{2^{n}} f$ converge to $f$ at any Lebesgue point $x$. Moreover, some new related inequalities are derived.

MSC: $26015 ; 42 \mathrm{C} 10 ; 42 \mathrm{~B} 30$

Keywords: Walsh system; Logarithmic means; Partial sums; Fejér means; Martingale Hardy space; Convergence; Divergence; Inequalities

\section{Abstract}

\section{Introduction}

The terminology and notations used in this introduction can be found in Sect. 2.

It is well known that Vilenkin systems do not form bases in the space $L_{1}$. Moreover, there is a function in the Hardy space $H_{1}$, such that the partial sums of $f$ are not bounded in the $L_{1}$-norm. Moreover, (see Tephnadze [22]) there exists a martingale $f \in H_{p}(0<p<1)$, such that

$$
\sup _{n \in \mathbb{N}}\left\|S_{2^{n}+1} f\right\|_{\text {weak }-L_{p}}=\infty
$$

On the other hand, (for details see, e.g., the books [20] and [25]) the subsequence $\left\{S_{2^{n}}\right\}$ of partial sums is bounded from the martingale Hardy space $H_{p}$ to the space $H_{p}$, for all $p>0$, that is, the following inequality holds:

$$
\left\|S_{2^{n}} f\right\|_{H_{p}} \leq c_{p}\|f\|_{H_{p}}, \quad n \in \mathbb{N}, p>0
$$

It is also well known that (see [20] and [16])

$$
S_{2^{n}} f(x) \rightarrow f(x), \quad \text { for all Lebesgue points of } f \in L_{p} \text {, where } p \geq 1
$$

(c) The Author(s) 2022. This article is licensed under a Creative Commons Attribution 4.0 International License, which permits use, sharing, adaptation, distribution and reproduction in any medium or format, as long as you give appropriate credit to the original author(s) and the source, provide a link to the Creative Commons licence, and indicate if changes were made. The images or other third party material in this article are included in the article's Creative Commons licence, unless indicated otherwise in a credit line to the material. If material is not included in the article's Creative Commons licence and your intended use is not permitted by statutory regulation or exceeds the permitted use, you will need to obtain permission directly from the copyright holder. To view a copy of this licence, visit http://creativecommons.org/licenses/by/4.0/. 
Weisz [26] considered the norm convergence of Fejér means of Vilenkin-Fourier series and proved that the inequality

$$
\left\|\sigma_{k} f\right\|_{p} \leq c_{p}\|f\|_{H_{p}}, \quad p>1 / 2 \text { and } f \in H_{p}
$$

holds. Moreover, Goginava [8] (see also $[12-15,18]$ ) proved that the assumption $p>1 / 2$ in (3) is essential. In particular, he showed that there exists a martingale $f \in H_{1 / 2}$ such that $\sup _{n \in \mathbb{N}}\left\|\sigma_{n} f\right\|_{1 / 2}=+\infty$. However, Weisz [26] (see also [17]) proved that for every $f \in H_{p}$, there exists an absolute constant $c_{p}$, such that the following inequality holds:

$$
\left\|\sigma_{2^{n}} f\right\|_{H_{p}} \leq c_{p}\|f\|_{H_{p}}, \quad n \in \mathbb{N}, p>0 .
$$

Móricz and Siddiqi [11] investigated the approximation properties of some special Nörlund means of Walsh-Fourier series of $L_{p}$ functions in norm. Approximation properties for general summability methods can be found in $[2,3]$. Fridli, Manchanda and Siddiqi [5] improved and extended the results of Móricz and Siddiqi [11] to martingale Hardy spaces. The case when $\left\{q_{k}=1 / k: k \in \mathbb{N}\right\}$ was excluded, since the methods are not applicable to Nörlund logarithmic means. In [6] Gát and Goginava proved some convergence and divergence properties of the Nörlund logarithmic means of functions in the Lebesgue space $L_{1}$. In particular, they proved that there exists a function in the space $L_{1}$, such that $\sup _{n \in \mathbb{N}}\left\|L_{n} f\right\|_{1}=\infty$.

In [4] (see also [10]) it was proved that there exists a martingale $f \in H_{p},(0<p<1)$ such that $\sup _{n \in \mathbb{N}}\left\|L_{n} f\right\|_{p}=\infty$.

In [19] (see also [24]) it was proved that there exists a martingale $f \in H_{1}$ such that

$$
\sup _{n \in \mathbb{N}}\left\|L_{n} f\right\|_{1}=\infty
$$

However, Goginava [7] proved that

$$
\left\|L_{2^{n} f}\right\|_{1} \leq c\|f\|_{1}, \quad f \in L_{1}, n \in \mathbb{N} .
$$

From this result it immediately follows that for every $f \in H_{1}$, there exists an absolute constant $c$, such that the inequality

$$
\left\|L_{2^{n}} f\right\|_{1} \leq c\|f\|_{H_{1}}
$$

holds for all $n \in \mathbb{N}$. Goginava [7] also proved that for any $f \in L_{1}(G)$,

$$
L_{2^{n}} f(x) \rightarrow f(x), \quad \text { a.e., as } n \rightarrow \infty .
$$

According to (1), (4) and (6), the following question is quite natural.

Question 1 Is the subsequence $\left\{L_{2^{n}}\right\}$ also bounded on the martingale Hardy spaces $H_{p}(G)$ when $0<p<1$ ? 
In Theorem 2 of this paper we give a negative answer to this question. In particular, we further develop some methods considered in $[1,9]$ and prove that for any $0<p<1$, there exists a martingale $f \in H_{p}$ such that $\sup _{n \in \mathbb{N}}\left\|L_{2^{n}} f\right\|_{\text {weak- } L_{p}}=\infty$. Moreover, in our Theorem 1 we generalize the result of Goginava [7] and prove that for any $f \in L_{1}(G)$ and for any Lebesgue point $x$,

$$
L_{2^{n}} f(x) \rightarrow f(x), \quad \text { as } n \rightarrow \infty .
$$

The main results in this paper are presented and proved in Sect. 4. Section 3 is used to present some auxiliary lemmas, where, in particular, Lemma 2 is new and of independent interest. In order not to disturb our discussions later some definitions and notations are given in Sect. 4. Finally, Sect. 5 is reserved for some open questions we hope can be a source of inspiration for further research in this interesting area.

\section{Definitions and notations}

Let $\mathbb{N}_{+}$denote the set of the positive integers, $\mathbb{N}:=\mathbb{N}_{+} \cup\{0\}$. Denote by $Z_{2}$ the discrete cyclic group of order 2 , that is $Z_{2}:=\{0,1\}$, where the group operation is the modulo 2 addition and every subset is open. The Haar measure on $Z_{2}$ is given so that the measure of a singleton is $1 / 2$.

Define the group $G$ as the complete direct product of the group $Z_{2}$, with the product of the discrete topologies of $Z_{2}$ s. The elements of $G$ are represented by sequences $x:=$ $\left(x_{0}, x_{1}, \ldots, x_{j}, \ldots\right)$, where $x_{k}=0 \vee 1$.

It is easy to give a base for the neighborhood of $x \in G$, namely:

$$
I_{0}(x):=G, \quad I_{n}(x):=\left\{y \in G: y_{0}=x_{0}, \ldots, y_{n-1}=x_{n-1}\right\} \quad(n \in \mathbb{N}) .
$$

Denote $I_{n}:=I_{n}(0), \overline{I_{n}}:=G \backslash I_{n}$ and $e_{n}:=\left(0, \ldots, 0, x_{n}=1,0, \ldots\right) \in G$, for $n \in \mathbb{N}$. It is easy to show that $\overline{I_{M}}=\bigcup_{s=0}^{M-1} I_{s} \backslash I_{s+1}$.

If $n \in \mathbb{N}$, then every $n$ can be uniquely expressed as $n=\sum_{k=0}^{\infty} n_{j} 2^{j}$, where $n_{j} \in Z_{2}(j \in \mathbb{N})$ and only a finite number of $n_{j}$ differ from zero. Let $|n|:=\max \left\{k \in \mathbb{N}: n_{k} \neq 0\right\}$.

The norms (or quasinorms) of the spaces $L_{p}(G)$ and weak $-L_{p}(G),(0<p<\infty)$ are, respectively, defined by

$$
\|f\|_{p}^{p}:=\int_{G}|f|^{p} d \mu, \quad\|f\|_{\text {weak-L }}^{p}:=\sup _{\lambda>0} \lambda^{p} \mu \quad(f>\lambda) .
$$

The $k$ th Rademacher function is defined by

$$
r_{k}(x):=(-1)^{x_{k}} \quad(x \in G, k \in \mathbb{N}) .
$$

Now, define the Walsh system $w:=\left(w_{n}: n \in \mathbb{N}\right)$ on $G$ as:

$$
w_{n}(x):=\prod_{k=0}^{\infty} r_{k}^{n_{k}}(x)=r_{|n|}(x)(-1)^{\sum_{k=0}^{|n|-1} n_{k} x_{k}} \quad(n \in \mathbb{N}) .
$$

It is well known that (see, e.g., [20])

$$
w_{n}(x+y)=w_{n}(x) w_{n}(y) .
$$


The Walsh system is orthonormal and complete in $L_{2}(G)$ (see, e.g., [20]).

If $f \in L_{1}(G)$ let us define Fourier coefficients, partial sums and the Dirichlet kernel by

$$
\begin{aligned}
& \widehat{f}(k):=\int_{G} f w_{k} d \mu \quad(k \in \mathbb{N}), \\
& S_{n} f:=\sum_{k=0}^{n-1} \widehat{f}(k) w_{k}, \quad D_{n}:=\sum_{k=0}^{n-1} w_{k} \quad\left(n \in \mathbb{N}_{+}\right) .
\end{aligned}
$$

Recall that (for details see, e.g., [20]):

$$
D_{2^{n}}(x)= \begin{cases}2^{n}, & \text { if } x \in I_{n}, \\ 0, & \text { if } x \notin I_{n},\end{cases}
$$

and

$$
D_{n}=w_{n} \sum_{k=0}^{\infty} n_{k} r_{k} D_{2^{k}}=w_{n} \sum_{k=0}^{\infty} n_{k}\left(D_{2^{k+1}}-D_{2^{k}}\right), \quad \text { for } n=\sum_{i=0}^{\infty} n_{i} 2^{i} .
$$

Let $\left\{q_{k}: k \geq 0\right\}$ be a sequence of nonnegative numbers. The Nörlund means for the Fourier series of $f$ are defined by

$$
t_{n} f:=\frac{1}{l_{n}} \sum_{k=0}^{n} q_{n-k} S_{k} f
$$

In the special case when $\left\{q_{k}=1: k \in \mathbb{N}\right\}$, we obtain the Fejér means

$$
\sigma_{n} f:=\frac{1}{n} \sum_{k=1}^{n} S_{k} f
$$

If $q_{k}=1 /(k+1)$, then we obtain the Nörlund logarithmic means:

$$
L_{n} f:=\frac{1}{l_{n}} \sum_{k=0}^{n-1} \frac{S_{k} f}{n-k}, \quad l_{n}:=\sum_{k=1}^{n} \frac{1}{k} .
$$

The Riesz logarithmic means are defined by

$$
R_{n} f:=\frac{1}{l_{n}} \sum_{k=1}^{n} \frac{S_{k} f}{k}, \quad l_{n}:=\sum_{k=1}^{n} \frac{1}{k}
$$

We note that this is an inverse of the Nörlund logarithmic means.

The convolution of two functions $f, g \in L_{1}(G)$ is defined by

$$
(f * g)(x):=\int_{G} f(x+t) g(t) d \mu(t) \quad(x \in G) .
$$

It is well known that if $f \in L_{p}(G), g \in L_{1}(G)$ and $1 \leq p<\infty$. Then, $f * g \in L_{p}(G)$ and the corresponding inequality holds:

$$
\|f * g\|_{p} \leq\|f\|_{p}\|g\|_{1} .
$$


The representations

$$
L_{n} f(x)=\int_{G} f(t) P_{n}(x+t) d \mu(t) \quad \text { and } \quad R_{n} f(x)=\int_{G} f(t) Y_{n}(x+t) d \mu(t)
$$

for $n \in \mathbb{N}$ play a central role in the following, where

$$
P_{n}:=\frac{1}{Q_{n}} \sum_{k=1}^{n} q_{n-k} D_{k} \quad \text { and } \quad Y_{n}:=\frac{1}{Q_{n}} \sum_{k=1}^{n} q_{k} D_{k}
$$

are called the kernels of the Nörlund logaritmic and the Reisz means, respectively. It is well known that (see, e.g., Goginava [7] and Tephnadze [23]):

$$
P_{2^{n}}(x)=D_{2^{n}}(x)-\psi_{2^{n}-1}(x) Y_{2^{n}}(x) .
$$

Moreover, for all $n \in \mathbb{N}$,

$$
\left\|P_{2^{n}}\right\|_{1}<c<\infty \text { and }\left\|Y_{n}\right\|_{1}<c<\infty .
$$

In the case $f \in L_{1}(G)$ the maximal functions are given by

$$
M(f)(x)=\sup _{n \in \mathbb{N}} \frac{1}{\left|I_{n}(x)\right|}\left|\int_{I_{n}(x)} f(u) d \mu(u)\right|=\sup _{n \in \mathbb{N}} 2^{n}\left|\int_{I_{n}(x)} f(u) d \mu(u)\right| .
$$

It is well known (for details see, e.g., [20]) that if $f \in L_{1}(G)$, then

$$
\|M(f)\|_{\text {weak- } L_{1}} \leq\|f\|_{1}
$$

According to a density argument of Calderon-Zygmund (see [20]) we obtain that if $f \in$ $L_{1}(G)$, then

$$
2^{n}\left|\int_{I_{n}(x)} f(u) d \mu(u)\right| \rightarrow 0, \quad \text { as } n \rightarrow \infty .
$$

A point $x$ on the Walsh group is called a Lebesgue point of $f \in L_{1}(G)$, if

$$
\lim _{n \rightarrow \infty} 2^{n} \int_{I_{n}(x)} f(t) d \mu(t)=f(x) \quad \text { a.e. } x \in G
$$

According to (2) we find that if $f \in L_{1}(G)$, then a.e. point is a Lebesgue point.

Let $f:=\left(f^{(n)}, n \in \mathbb{N}\right)$ be a martingale with respect to $\digamma_{n}(n \in \mathbb{N})$, which are generated by the intervals $\left\{I_{n}(x): x \in G\right\}$ (for details see, e.g., [25]).

We say that a martingale belongs to Hardy martingale spaces $H_{p}(G)$, where $0<p<\infty$ if $\|f\|_{H_{p}}:=\left\|f^{*}\right\|_{p}<\infty$, where $f^{*}:=\sup _{n \in \mathbb{N}}\left|f^{(n)}\right|$.

If $f=\left(f^{(n)}, n \in \mathbb{N}\right)$ is a martingale, then the Walsh-Fourier coefficients must be defined in a slightly different manner:

$$
\widehat{f}(i):=\lim _{k \rightarrow \infty} \int_{G} f^{(k)}(x) w_{i}(x) d \mu(x) .
$$




\section{Auxiliary results}

The Hardy martingale space $H_{p}(G)$ has an atomic characterization (see Weisz $[25,26]$ ).

Lemma 1 A martingale $f=\left(f^{(n)}, n \in \mathbb{N}\right)$ is in $H_{p}(0<p \leq 1)$ if and only if there exist a sequence $\left(a_{k}, k \in \mathbb{N}\right)$ of $p$-atoms, which means that they satisfy the conditions

$$
\int_{I} a_{k} d \mu=0, \quad\left\|a_{k}\right\|_{\infty} \leq \mu(I)^{-1 / p}, \quad \operatorname{supp}\left(a_{k}\right) \subset I,
$$

and a sequence $\left(\mu_{k}, k \in \mathbb{N}\right)$ of real numbers such that for every $n \in \mathbb{N}$ :

$$
\sum_{k=0}^{\infty} \mu_{k} S_{2^{n}} a_{k}=f^{(n)}, \quad \text { where } \sum_{k=0}^{\infty}\left|\mu_{k}\right|^{p}<\infty .
$$

Moreover, $\|f\|_{H_{p}} \backsim \inf \left(\sum_{k=0}^{\infty}\left|\mu_{k}\right|^{p}\right)^{1 / p}$, where the infimum is taken over all decompositions off of the form (14).

We also state and prove a new lemma of independent interest:

Lemma 2 Let $n \in \mathbb{N}$ and $x \in I_{2}\left(e_{0}+e_{1}\right) \in I_{0} \backslash I_{1}$. Then,

$$
\left|\sum_{j=2^{2 \alpha_{k}}}^{2 \alpha_{k}+1} \frac{D_{j}}{2^{2 \alpha_{k}+1}-j}\right| \geq \frac{1}{3}
$$

Proof Let $x \in I_{2}\left(e_{0}+e_{1}\right) \in I_{0} \backslash I_{1}$. According to (8) and (9) we obtain that

$$
D_{j}(x)= \begin{cases}w_{j}, & \text { if } j \text { is an odd number, } \\ 0, & \text { if } j \text { is an even number }\end{cases}
$$

and

$$
\sum_{j=2^{2 \alpha_{k}}}^{2^{2 \alpha_{k}+1}-1} \frac{D_{j}}{2^{2 \alpha_{k}+1}-j}=\sum_{j=2^{2 \alpha_{k}-1}}^{2 \alpha_{k}-1} \frac{w_{2 j+1}}{2^{2 \alpha_{k}+1}-2 j-1}=w_{1} \sum_{j=2^{2 \alpha_{k}-1}}^{2^{2 \alpha_{k}-1}} \frac{w_{2 j}}{2^{2 \alpha_{k}+1}-2 j-1} .
$$

Since

$$
\begin{aligned}
& \sum_{j=2^{2 \alpha_{k}-2}+1}^{2^{2 \alpha_{k}-1}-1}\left|\frac{1}{2^{2 \alpha_{k}+1}-4 j+3}-\frac{1}{2^{2 \alpha_{k}+1}-4 j+1}\right| \\
& =\sum_{j=2^{2 \alpha_{k}-2}+1}^{2^{2 \alpha_{k}-1}-1} \frac{2}{\left(2^{2 \alpha_{k}+1}-4 j+3\right)\left(2^{2 \alpha_{k}+1}-4 j+1\right)} \\
& \leq \sum_{j=2^{2 \alpha_{k}-2}+1}^{2^{2 \alpha_{k}-1}-1} \frac{2}{\left(2^{2 \alpha_{k}+1}-4 j\right)\left(2^{2 \alpha_{k}+1}-4 j\right)} \\
& \leq \frac{1}{8} \sum_{j=2^{2 \alpha_{k}-2}+1}^{2^{2 \alpha_{k}-1}-1} \frac{1}{\left(2 \alpha^{2 \alpha_{k}-1}-j\right)\left(2^{2 \alpha_{k}-1}-j\right)}
\end{aligned}
$$




$$
\begin{aligned}
& \leq \frac{1}{8} \sum_{k=1}^{\infty} \frac{1}{k^{2}} \leq \frac{1}{8}+\frac{1}{8} \sum_{k=2}^{\infty} \frac{1}{k^{2}} \\
& \leq \frac{1}{8}+\frac{1}{8} \sum_{k=2}^{\infty}\left(\frac{1}{k-1}-\frac{1}{k}\right) \\
& \leq \frac{1}{8}+\frac{1}{8}=\frac{1}{4},
\end{aligned}
$$

if we apply $w_{4 k+2}=w_{2} w_{4 k}=-w_{4 k}$, for $x \in I_{2}\left(e_{0}+e_{1}\right)$, we find that

$$
\begin{aligned}
\left|\sum_{j=2^{2 \alpha_{k}}}^{2 \alpha_{k}+1} \frac{D_{j}}{2^{2 \alpha_{k}+1}-j}\right| & =\left|w_{2^{2 \alpha_{k}+1}-2}+\frac{w_{2^{2 \alpha_{k}+1}-4}}{3}+\sum_{j=2^{2 \alpha_{k}-1}+1}^{2^{2 \alpha_{k}-1}} \frac{w_{2 j}}{2^{2 \alpha_{k}+1}-2 j-1}\right| \\
& =\left|\frac{w_{2^{2 \alpha_{k}+1}-4}}{3}-w_{2^{2 \alpha_{k}+1}-4}+\sum_{j=2^{2 \alpha_{k}-1}+1}^{2^{2 \alpha_{k}-1}} \frac{w_{2 j}}{2^{2 \alpha_{k}+1}-2 j-1}\right| \\
& =\left|\frac{2 w_{2^{2 \alpha_{k}+1}-4}}{3}+\sum_{j=2^{2 \alpha_{k}-2}+1}^{2^{2 \alpha_{k}-1}}\left(\frac{w_{4 j-4}}{2^{2 \alpha_{k}+1}-4 j+3}+\frac{w_{4 j-2}}{2^{2 \alpha_{k}+1}-4 j+1}\right)\right| \\
& =\left|\frac{2 w_{2^{2 \alpha_{k}+1}-4}}{3}+\sum_{j=2^{2 \alpha_{k}-2}+1}^{2^{2 \alpha_{k}-1}}\left(\frac{w_{4 j-4}}{2^{2 \alpha_{k}+1}-4 j+3}-\frac{w_{4 j-4}}{2^{2 \alpha_{k}+1}-4 j+1}\right)\right| \\
& \geq \frac{2}{3}-\sum_{j=2^{2 \alpha_{k}-2}+1}\left|\frac{1}{2^{2 \alpha_{k}+1}-4 j+3}-\frac{1}{2^{2 \alpha_{k}+1}-4 j+1}\right| \\
& \geq \frac{2}{3}-\frac{1}{4} \geq \frac{1}{3} .
\end{aligned}
$$

The proof is complete.

\section{Main results}

Our first main result reads:

Theorem 1 Let $p \geq 1$ and $f \in L_{p}(G)$. Then,

$$
\left\|L_{2^{n}} f-f\right\|_{p} \rightarrow 0 \quad \text { as } n \rightarrow \infty .
$$

Moreover, for all Lebesgue points of $f$,

$$
\lim _{n \rightarrow \infty} L_{2^{n}} f(x)=f(x)
$$

Proof Let $n \in \mathbb{N}$. By combining (11) and (13) we immediately obtain

$$
\left\|L_{2^{n} f}\right\|_{p} \leq c_{p}\|f\|_{p} \quad \text { for all } n \in \mathbb{N}
$$

which immediately implies (15). 
To prove a.e. convergence we use identity (12) to obtain that

$$
\begin{aligned}
L_{2^{n}} f(x)= & \int_{G} f(t) P_{2^{n}}(x+t)=\int_{G} f(t) D_{2^{n}}(x+t) d \mu(t) d \mu(t) \\
& -\int_{G} f(t) w_{2^{n}-1}(x+t) Y_{2^{n}}(x+t) d \mu(t):=I-I I .
\end{aligned}
$$

By applying (2) we can conclude that $I=S_{2^{n}} f(x) \rightarrow f(x)$ for all Lebesgue points of $f \in L_{p}$. Moreover, by using (7) we find that

$$
I I=\psi_{2^{n}-1}(x) \int_{G} f(t) Y_{2^{n}}(x+t) \psi_{2^{n}-1}(t) d(t) .
$$

In view of (13) we see that

$$
f(t) Y_{2^{n}}(x+t) \in L_{p} \quad \text { where } p \geq 1 \text { for any } x \in G,
$$

and also note that $I I$ describes the Fourier coefficients of an integrable function. Hence, according to the Riemann-Lebesgue Lemma it vanishes as $n \rightarrow \infty$, i.e., $I I \rightarrow 0$ for any $x \in G, n \rightarrow \infty$.

The proof is complete.

Our next main result is the following answer of Question 1.

Theorem 2 Let $0<p<1$. Then, there exists a martingale $f \in H_{p}$ such that

$$
\sup _{n \in \mathbb{N}}\left\|L_{2^{n}} f\right\|_{\text {weak }-L_{p}}=\infty .
$$

Proof Let $\left\{\alpha_{k}: k \in \mathbb{N}\right\}$ be an increasing sequence of the positive integers such that

$$
\begin{aligned}
& \sum_{k=0}^{\infty} \alpha_{k}^{-p / 2}<\infty \\
& \sum_{\eta=0}^{k-1} \frac{\left(2^{2 \alpha_{\eta}}\right)^{1 / p}}{\sqrt{\alpha_{\eta}}}<\frac{\left(2^{2 \alpha_{k}}\right)^{1 / p}}{\sqrt{\alpha_{k}}},
\end{aligned}
$$

and

$$
\frac{\left(2^{2 \alpha_{k-1}}\right)^{1 / p}}{\sqrt{\alpha_{k-1}}}<\frac{2^{2 \alpha_{k}-8}}{\alpha_{k}^{1 / 2} l_{2^{2 \alpha_{k}+1}}} .
$$

Let

$$
f^{(n)}(x):=\sum_{\left\{k ; 2 \alpha_{k}<n\right\}} \lambda_{k} a_{k}
$$

where

$$
\lambda_{k}=\frac{1}{\sqrt{\alpha_{k}}} \quad \text { and } \quad a_{k}=2^{2 \alpha_{k}(1 / p-1)}\left(D_{2^{2 \alpha_{k}+1}}-D_{2^{2 \alpha_{k}}}\right) .
$$


From (16) and Lemma 1 we find that $f \in H_{p}$. It is easy to show that

$$
\widehat{f}(j)= \begin{cases}\frac{2^{2 \alpha_{k}(1 / p-1)}}{\sqrt{\alpha_{k}}}, & \text { if } j \in\left\{2^{2 \alpha_{k}}, \ldots, 2^{2 \alpha_{k}+1}-1\right\}, k \in \mathbb{N} \\ 0, & \text { if } j \notin \bigcup_{k=1}^{\infty}\left\{2^{2 \alpha_{k}}, \ldots, 2^{2 \alpha_{k}+1}-1\right\} .\end{cases}
$$

Moreover,

$$
\begin{aligned}
L_{2^{2 \alpha_{k}+1}} f & =\frac{1}{l_{2^{2 \alpha_{k}+1}}} \sum_{j=1}^{2 \alpha_{k}-1} \frac{S_{j} f}{2^{2 \alpha_{k}+1}-j}+\frac{1}{l_{2^{2 \alpha_{k}+1}}} \sum_{j=2^{2 \alpha_{k}}}^{2 \alpha_{k}+1} \frac{S_{j} f}{2^{2 \alpha_{k}+1}-j} \\
& :=I+I I .
\end{aligned}
$$

Let $j<2^{2 \alpha_{k}}$. By combining (17), (18) and (19) we can conclude that

$$
\begin{aligned}
\left|S_{j} f(x)\right| & \leq \sum_{\eta=0}^{k-1} \sum_{v=2^{2 \alpha_{\eta}}}^{2^{2 \alpha_{\eta}+1}-1}|\widehat{f}(v)| \\
& \leq \sum_{\eta=0}^{k-1} \sum_{\nu=2^{2 \alpha_{\eta}}}^{2^{2 \alpha_{\eta}+1}-1} \frac{2^{2 \alpha_{\eta}(1 / p-1)}}{\sqrt{\alpha_{\eta}}} \\
& \leq \sum_{\eta=0}^{k-1} \frac{2^{2 \alpha_{\eta} / p}}{\sqrt{\alpha_{\eta}}} \\
& \leq \frac{2^{2 \alpha_{k-1} / p+1}}{\sqrt{\alpha_{k-1}}}<\frac{2^{2 \alpha_{k}-4}}{\alpha_{k}^{1 / 2} l_{2^{2 \alpha_{k}+1}}} .
\end{aligned}
$$

Hence,

$$
\begin{aligned}
|I| & \leq \frac{1}{l_{2^{2 \alpha_{k}+1}}} \sum_{j=1}^{2^{2 \alpha_{k}-1}} \frac{\left|S_{j} f(x)\right|}{2^{2 \alpha_{k}+1}-j} \\
& \leq \frac{1}{l_{2^{2 \alpha_{k}+1}}} \frac{2^{2 \alpha_{k-1} / p}}{\sqrt{\alpha_{k-1}}} \sum_{j=1}^{M_{2 \alpha_{k}+1}-1} \frac{1}{j} \\
& \leq \frac{2^{2 \alpha_{k-1} / p}}{\sqrt{\alpha_{k-1}}} .
\end{aligned}
$$

Let $2^{2 \alpha_{k}} \leq j \leq 2^{2 \alpha_{k}+1}-1$. We can write that

$$
\begin{aligned}
S_{j} f & =\sum_{\eta=0}^{k-1} \sum_{\nu=2^{2 \alpha_{\eta}}}^{2^{2 \alpha_{\eta}+1}-1} \widehat{f}(v) w_{v}+\sum_{v=2^{2 \alpha_{k}}}^{j-1} \widehat{f}(v) w_{v} \\
& =\sum_{\eta=0}^{k-1} \frac{2^{2 \alpha_{\eta}(1 / p-1)}}{\sqrt{\alpha_{\eta}}}\left(D_{2^{2 \alpha_{\eta}+1}}-D_{2^{2 \alpha_{\eta}}}\right)+\frac{2^{2 \alpha_{k}(1 / p-1)}}{\sqrt{\alpha_{k}}}\left(D_{j}-D_{2^{2 \alpha_{k}}}\right) .
\end{aligned}
$$


It follows that

$$
\begin{aligned}
I I= & \frac{1}{l_{2^{2 \alpha_{k}+1}}} \sum_{j=2^{2 \alpha_{k}}}^{2^{2 \alpha_{k}+1}} \frac{1}{2^{2 \alpha_{k}+1}-j}\left(\sum_{\eta=0}^{k-1} \frac{2^{2 \alpha_{\eta}(1 / p-1)}}{\sqrt{\alpha_{\eta}}}\left(D_{2^{2 \alpha_{\eta}+1}}-D_{2^{2 \alpha_{\eta}}}\right)\right) \\
& +\frac{1}{l_{2^{2 \alpha_{k}+1}}} \frac{2^{2 \alpha_{k}(1 / p-1)}}{\sqrt{\alpha_{k}}} \sum_{j=2^{2 \alpha_{k}}}^{2^{2 \alpha_{k}+1}-1} \frac{\left(D_{j}-D_{2^{2 \alpha_{k}}}\right)}{2^{2 \alpha_{k}+1}-j} \\
:= & I I_{1}+I I_{2} .
\end{aligned}
$$

Let $x \in I_{2}\left(e_{0}+e_{1}\right) \in I_{0} \backslash I_{1}$. According to $\alpha_{0} \geq 1$ we obtain that $2 \alpha_{k} \geq 2$, for all $k \in \mathbb{N}$ and if we use (8) we obtain that $D_{2^{2 \alpha_{k}}}=0$,

$$
I I_{1}=0
$$

and

$$
\begin{aligned}
I_{2} & =\frac{1}{l_{2^{2 \alpha_{k}+1}}} \frac{2^{2 \alpha_{k}(1 / p-1)}}{\sqrt{\alpha_{k}}} \sum_{j=2^{2 \alpha_{k}-1}+1}^{2^{2 \alpha_{k}-1}} \frac{w_{2 j+1}}{2^{2 \alpha_{k}+1}-2 j-1} \\
& =\frac{1}{l_{2^{2 \alpha_{k}+1}}} \frac{2^{2 \alpha_{k}(1 / p-1)} w_{1}}{\sqrt{\alpha_{k}}} \sum_{j=2^{2 \alpha_{k}-1}+1}^{2^{2 \alpha_{k}-1}} \frac{w_{2 j}}{2^{2 \alpha_{k}+1}-2 j-1} .
\end{aligned}
$$

By using Lemma 2 we can conclude that

$$
\left|I I_{2}\right| \geq \frac{1}{3} \frac{1}{l_{2^{2 \alpha_{k}+1}}} \frac{2^{2 \alpha_{k}(1 / p-1)}}{\sqrt{\alpha_{k}}} \geq \frac{1}{l_{2^{2 \alpha_{k}+1}}} \frac{2^{2 \alpha_{k}(1 / p-1)-1}}{\sqrt{\alpha_{k}}} .
$$

If we apply (18), (20)-(24) for $x \in I_{2}\left(e_{0}+e_{1}\right)$ and $0<p<1$, we have that

$$
\begin{aligned}
\left|L_{2^{2 \alpha_{k}+1}} f(x)\right| & \geq I I_{2}-I I_{1}-I \\
& \geq \frac{1}{l_{2^{2 \alpha_{k}+1}}} \frac{2^{2 \alpha_{k}(1 / p-1)-2}}{\sqrt{\alpha_{k}}}-\frac{1}{l_{2^{2 \alpha_{k}+1}}} \frac{2^{2 \alpha_{k}(1 / p-1)-3}}{\sqrt{\alpha_{k}}} \\
& \geq \frac{1}{l_{2^{2 \alpha_{k}+1}}} \frac{2^{2 \alpha_{k}(1 / p-1)-3}}{\sqrt{\alpha_{k}}} \\
& \geq \frac{2^{2 \alpha_{k}(1 / p-1)-3}}{\left(\ln 2^{2 \alpha_{k}+1}+1\right) \sqrt{\alpha_{k}}} \\
& \geq \frac{2^{2 \alpha_{k}(1 / p-1)-3}}{\left(4 \alpha_{k}+1\right) \sqrt{\alpha_{k}}} \geq \frac{2^{2 \alpha_{k}(1 / p-1)-6}}{\alpha_{k}^{3 / 2}} .
\end{aligned}
$$

Hence, we can conclude that

$$
\begin{aligned}
& \left\|L_{q_{\alpha_{k}}^{s}} f\right\|_{\text {weak- } L_{p}} \\
& \quad \geq \frac{2^{2 \alpha_{k}(1 / p-1)-6}}{\alpha_{k}^{3 / 2}} \mu\left\{x \in G:\left|L_{2^{2 \alpha_{k}+1}} f\right| \geq \frac{2^{2 \alpha_{k}(1 / p-1)-6}}{\alpha_{k}^{3 / 2}}\right\}^{1 / p}
\end{aligned}
$$




$$
\begin{aligned}
& \geq \frac{2^{2 \alpha_{k}(1 / p-1)-6}}{\alpha_{k}^{3 / 2}} \mu\left\{x \in I_{2}\left(e_{0}+e_{1}\right):\left|L_{2^{2 \alpha_{k}+1}} f\right| \geq \frac{2^{2 \alpha_{k}(1 / p-1)-6}}{\alpha_{k}^{3 / 2}}\right\}^{1 / p} \\
& \geq \frac{2^{2 \alpha_{k}(1 / p-1)-6}}{\alpha_{k}^{3 / 2}}\left(\mu\left(I_{2}\left(e_{0}+e_{1}\right)\right)\right)^{1 / p} \\
& >\frac{c 2^{2 \alpha_{k}(1 / p-1)}}{\alpha_{k}^{3 / 2}} \rightarrow \infty, \quad \text { as } k \rightarrow \infty .
\end{aligned}
$$

The proof is complete.

\section{Open questions}

It is known (for details see, e.g., the books [20] and [25]) that the subsequence $\left\{S_{2^{n}}\right\}$ of the partial sums is bounded from the martingale Hardy space $H_{p}$ to the Lebesgue space $L_{p}$, for all $p>0$. On the other hand, (see Tephnadze [22]) there exists a martingale $f \in H_{p}$ $(0<p<1)$, such that $\sup _{n \in \mathbb{N}}\left\|S_{2^{n}+1} f\right\|_{\text {weak }-L_{p}}=\infty$. However, Simon [21] proved that for all $f \in H_{p}$, there exists an absolute constant $c_{p}$, depending only on $p$, such that

$$
\sum_{k=1}^{\infty} \frac{\left\|S_{k} f\right\|_{p}^{p}}{k^{2-p}} \leq c_{p}\|f\|_{H_{p}}^{p}, \quad(0<p<1)
$$

In [24] it was proved that for all $f \in H_{p}$, there exists an absolute constant $c_{p}$, depending only on $p$, such that

$$
\sum_{k=1}^{\infty} \frac{\left\|L_{k} f\right\|_{p}^{p}}{k^{2-p}} \leq c_{p}\|f\|_{H_{p}}^{p}, \quad(0<p<1)
$$

Open Problem 1 (a) Let $f \in H_{p}$, where $0<p<1$. Does there exist an absolute constant $c_{p}$, such that the following inequality holds:

$$
\sum_{k=1}^{\infty} \frac{\log ^{p} k\left\|L_{k} f\right\|_{p}^{p}}{k^{2-p}} \leq c_{p}\|f\|_{H_{p}}^{p}, \quad(0<p<1) ?
$$

(b) For $0<p<1 / 2$ and any nondecreasing function $\Phi: \mathbb{N} \rightarrow[1, \infty)$ satisfying the conditions $\lim _{n \rightarrow \infty} \Phi(n)=+\infty$, is it possible to find a martingale $f \in H_{p}$ such that

$$
\sum_{n=1}^{\infty} \frac{\log ^{p} n\left\|L_{n} f\right\|_{p}^{p} \Phi(n)}{n^{2-p}}=\infty ?
$$

Open Problem 2 (a) Let $f \in H_{p}$, where $0<p \leq 1$ and

$$
\omega_{H_{p}}\left(\frac{1}{2^{n}}, f\right)=o\left(\frac{\log n}{2^{n(1 / p-1)} \log ^{2[p]} n}\right), \quad \text { as } n \rightarrow \infty .
$$

Does the following convergence result hold:

$$
\left\|L_{k} f-f\right\|_{H_{p}} \rightarrow 0, \quad \text { as } k \rightarrow \infty ?
$$


(b) Let $0<p \leq 1$. Does there exist a martingale $f \in H_{p}$, for which

$$
\omega_{H_{p}}\left(\frac{1}{2^{n}}, f\right)=O\left(\frac{\log n}{2^{n(1 / p-1)} \log ^{2[p]} n}\right), \quad \text { as } n \rightarrow \infty
$$

and $\left\|L_{k} f-f\right\|_{\text {weak }-L_{p}} \nrightarrow 0$, as $k \rightarrow \infty$ ?

\section{Acknowledgements}

The work of George Tephnadze was supported by the Shota Rustaveli National Science Foundation grant FR-19-676. The publication charges for this article have been funded by a grant from the publication fund of UiT The Arctic University of Norway. The authors also would like to thank the two referees for helpful suggestions.

\section{Funding}

The publication charges for this manuscript were supported by the publication fund at UiT The Arctic University of Norway under code IN-1096130. Open Access funding provided by UiT The Arctic University of Norway.

Availability of data and materials

Not applicable.

\section{Declarations}

\section{Competing interests}

The authors declare that they have no competing interests.

\section{Authors' contributions}

DB and GT proposed the idea and initiated the writing of this paper. LEP and HS followed this up with some complementary ideas. All the authors read and approved the final manuscript.

\section{Author details}

${ }^{1}$ School of Science and Technology, The University of Georgia, 77a Merab Kostava St, Tbilisi 0128, Georgia. ${ }^{2}$ UiT The Arctic University of Norway, P.O. Box 385, N-8505, Narvik, Norway. ${ }^{3}$ Department of Mathematics and Computer Science, Karlstad University, 65188 Karlstad, Sweden.

\section{Publisher's Note}

Springer Nature remains neutral with regard to jurisdictional claims in published maps and institutional affiliations.

Received: 8 September 2021 Accepted: 7 February 2022 Published online: 07 March 2022

\section{References}

1. Baramidze, L., Persson, L.E., Tephnadze, G., Wall, P.: Sharp $H_{p}-L_{p}$ type inequalities of weighted maximal operators of Vilenkin-Nörlund means and its applications. J. Inequal. Appl. (2016). https://doi.org/10.1186/s13660-016-1182-1

2. Blahota, I., Nagy, K., Tephnadze, G.: Approximation by $\Theta$-means of Walsh-Fourier series. Anal. Math. 44(1), 57-71 (2018)

3. Blahota, I., Nagy, K., Tephnadze, G.: Approximation by Marcinkiewicz $\Theta$-means of double Walsh-Fourier series. Math Inequal. Appl. 22(3), 837-853 (2019)

4. Blahota, I., Persson, L.-E., Tephnadze, G.: On the Nörlund means of Vilenkin-Fourier series. Czechoslov. Math. J. 65(4), 983-1002 (2015)

5. Fridli, S., Manchanda, P., Siddiqi, A.H.: Approximation by Walsh-Nörlund means. Acta Sci. Math. 74(3-4), 593-608 (2008)

6. Gàt, G., Goginava, U.: Uniform and L-convergence of logarithmic means of Walsh-Fourier series. Acta Math. Sin. 22(2), 497-506 (2006)

7. Goginava, U.: Almost everywhere convergence of subsequence of logarithmic means of Walsh-Fourier series. Acta Math. Acad. Paedagog. Nyházi. 21, 169-175 (2005)

8. Goginava, U.: The maximal operator of the $(C, \alpha)$ means of the Walsh-Fourier series. Ann. Univ. Sci. Bp. Sect. Comput. 26, 127-135 (2006)

9. Lukkassen, D., Persson, L.E., Tephnadze, G., Tutberidze, G.: Some inequalities related to strong convergence of Riesz logarithmic means of Vilenkin-Fourier series. J. Inequal. Appl. (2020). https://doi.org/10.1186/s13660-020-02342-8

10. Memić, N., Persson, L.E., Tephnadze, G.: A note on the maximal operators of Vilenkin-Nörlund means with non-increasing coefficients. Studia Sci. Math. Hung. 53(4), 545-556 (2016)

11. Móricz, F., Siddiqi, A.: Approximation by Nörlund means of Walsh-Fourier series. J. Approx. Theory 70(3), 375-389 (1992)

12. Nagy, K., Tephnadze, G.: On the Walsh-Marcinkiewicz means on the Hardy space. Cent. Eur. J. Math. 12(8), 1214-1228 (2014)

13. Nagy, K., Tephnadze, G.: Approximation by Walsh-Marcinkiewicz means on the Hardy space. Kyoto J. Math. 54(3), 641-652 (2014)

14. Nagy, K., Tephnadze, G.: Kaczmarz-Marcinkiewicz means and Hardy spaces. Acta Math. Hung. 149(2), 346-374 (2016) 
15. Nagy, K., Tephnadze, G.: Strong convergence theorem for Walsh-Marcinkiewicz means. Math. Inequal. Appl. 19(1), 185-195 (2016)

16. Persson, L.-E., Schipp, F., Tephnadze, G., Weisz, F.: An analogy of the Carleson-Hunt theorem with respect to Vilenkin systems. J. Fourier Anal. Appl. (to appear)

17. Persson, L.E., Tephnadze, G.: A sharp boundedness result concerning some maximal operators of Vilenkin-Fejér means. Mediterr. J. Math. 13(4), 1841-1853 (2016)

18. Persson, L.E., Tephnadze, G., Tutberidze, G.: On the boundedness of subsequences of Vilenkin-Fejér means on the martingale Hardy spaces. Oper. Matrices 14(1), 283-294 (2020)

19. Persson, L.E., Tephnadze, G., Wall, P.: On the Nörlund logarithmic means with respect to Vilenkin system in the martingale Hardy space $H_{1}$. Acta Math. Hung. 154(2), 289-301 (2018)

20. Schipp, F., Wade, W.R., Simon, P., Pál, J.: Walsh Series, an Introduction to Dyadic Harmonic Analysis. Akad. Kiadó, Budapest (1990)

21. Simon, P.: Strong convergence theorem for Vilenkin-Fourier series. J. Math. Anal. Appl. 245, 52-68 (2000)

22. Tephnadze, G.: On the partial sums of Vilenkin-Fourier series. J. Contemp. Math. Anal. 49(1), 23-32 (2014)

23. Tephnadze, G.: Martingale Hardy Spaces and Summability of the One Dimensional Vilenkin-Fourier Series. PhD thesis, Department of Engineering Sciences and Mathematics, LuleåUniversity of Technology (2015). (ISSN 1402-1544)

24. Tephnadze, G., Tutberidze, G.: A note on the maximal operators of the Nörlund logaritmic means of Vilenkin-Fourier series. Trans. A. Razmadze Math. Inst. 174(1), 1070-1112 (2020)

25. Weisz, F.: Martingale Hardy Spaces and Their Applications in Fourier Analysis. Springer, Berlin (1994)

26. Weisz, F.: Hardy spaces and Cesàro means of two-dimensional Fourier series. In: Bolyai Soc. Math. Studies, pp. 353-367 (1996)

\section{Submit your manuscript to a SpringerOpen ${ }^{\circ}$ journal and benefit from:}

- Convenient online submission

- Rigorous peer review

- Open access: articles freely available online

- High visibility within the field

- Retaining the copyright to your article

Submit your next manuscript at $\boldsymbol{~ s p r i n g e r o p e n . c o m ~}$ 